\title{
ALGEBRAIC CONVERGENCE OF SCHOTTKY GROUPS
}

\author{
RICHARD D. CANARY
}

\begin{abstract}
A discrete faithful representation of the free group on $g$ generators $F_{g}$ into $\operatorname{Isom}_{+}\left(\mathbf{H}^{3}\right)$ is said to be a Schottky group if $\left(\mathbf{H}^{3} \cup D_{\Gamma}\right) / \Gamma$ is homeomorphic to a handlebody $H_{g}$ (where $D_{\Gamma}$ is the domain of discontinuity for $\Gamma$ 's action on the sphere at infinity for $\mathbf{H}^{3}$ ). Schottky space $\mathscr{S}_{g}$, the space of all Schottky groups, is parameterized by the quotient of the Teichmüller space $\mathscr{T}\left(S_{g}\right)$ of the closed surface of genus $g$ by $\operatorname{Mod}_{0}\left(H_{g}\right)$ where $\operatorname{Mod}_{0}\left(H_{g}\right)$ is the group of (isotopy classes of) homeomorphisms of $S_{g}$ which extend to homeomorphisms of $H_{g}$ which are homotopic to the identity. Masur exhibited a domain $\mathscr{O}\left(H_{g}\right)$ of discontinuity for $\operatorname{Mod}_{0}\left(H_{g}\right)$ 's action on $P L\left(S_{g}\right)$ (the space of projective measured laminations on $\left.S_{g}\right)$, so $\mathscr{B}\left(H_{g}\right)=\mathscr{O}\left(H_{g}\right) / \operatorname{Mod}_{0}\left(H_{g}\right)$ may be appended to $\mathscr{S}_{g}$ as a boundary. Thurston conjectured that if a sequence $\left\{\rho_{i}: F_{g} \rightarrow\right.$ Isom $\left._{+}\left(\mathbf{H}^{3}\right)\right\}$ of Schottky groups converged into $\mathscr{B}\left(H_{g}\right)$, then it converged as a sequence of representations, up to subsequence and conjugation. In this paper, we prove Thurston's conjecture in the case where $H_{g}$ is homeomorphic to $S \times I$ and the length $l_{N_{i}}\left((\partial S)^{*}\right)$ in $N_{i}=\mathbf{H}^{3} / \rho_{i}\left(F_{g}\right)$ of the closed geodesic(s) in the homotopy class of the boundary of $S$ is bounded above by some constant $K$.
\end{abstract}

\section{INTRODUCTION}

The techniques developed in Thurston's proof of his geometrization theorem ([36, 37, 38], see also Ohshika [32]) give one a very good understanding of deformations of hyperbolic structures on 3-manifolds with incompressible boundary. In particular, one can often determine whether a sequence of convex cocompact structures converges algebraically by looking at the limiting behavior of their associated conformal structures at infinity. However, the theory for manifolds with compressible boundary is not so well understood. A theorem of Thurston's allows one to establish algebraic convergence of a sequence of hyperbolic structures if one knows that the lengths of some doubly incompressible (see below) system of curves is bounded, but this does not usually allow one to understand convergence by looking at the conformal structures at infinity. Handlebodies are the most basic examples of 3-manifolds with compressible boundary; in this paper we will investigate algebraic convergence of convex cocompact hyperbolic structures on handlebodies.

We will think of a Kleinian group as a discrete, faithful representation $\rho: G \rightarrow \mathrm{PSL}_{2}(\mathbf{C})$ with image $\Gamma$. $\mathrm{PSL}_{2}(\mathbf{C})$ may be identified, via the upper

Received by the editors February 1, 1991.

1980 Mathematics Subject Classification (1985 Revision). Primary 30F40, 32G15, 57M99, 58H15.

Partially supported by NSF grant DMS 88-09085. 
half-space model, with the group of orientation-preserving isometries of hyperbolic 3-space $\mathbf{H}^{3} . \mathbf{C} \cup\{\infty\}$, regarded as the sphere at infinity for $\mathbf{H}^{3}$, may be divided into the limit set $L_{\Gamma}$ for $\Gamma$ 's action on $\mathrm{C} \cup\{\infty\}$ and the domain of discontinuity $D_{\Gamma}$. A torsion-free Kleinian group $\Gamma$ is said to be a Schottky group of genus $g$ if $\left(\mathrm{H}^{3} \cup D_{\Gamma}\right) / \Gamma$ is homeomorphic to a handlebody $H_{g}$ of genus $g$. (See Maskit [25] for basic definitions in the theory of Kleinian groups.) Given a homeomorphism $h: H_{g} \rightarrow\left(\mathbf{H}^{3} \cup D_{\Gamma}\right) / \Gamma$ we may think of $\mathbf{H}^{3} / \Gamma$ as a hyperbolic structure on the interior of $H_{g}$ and $D_{\Gamma} / \Gamma$ as a conformal structure on the boundary of $H_{g}$.

We may think of $\rho: F_{g} \rightarrow \mathrm{PSL}_{2}(\mathbf{C})$ (where $F_{g}$ denotes the free group on $g$ generators) as an isomorphism of $\Gamma$ with $\pi_{1}\left(H_{g}\right)$. This isomorphism induces a homotopy equivalence $g: H_{g} \rightarrow\left(\mathbf{H}^{3} \cup D_{\Gamma}\right) / \Gamma . g$ is homotopic to many different homeomorphisms $h: H_{g} \rightarrow\left(\mathbf{H}^{3} \cup D_{\Gamma}\right) / \Gamma$. If we precompose by any homeomorphism $j: H_{g} \rightarrow H_{g}$ which is homotopic to the identity $h \circ j$ is homotopic to $g$. Therefore the conformal structure on $D_{\Gamma} / \Gamma$ is only well defined as an element of $\mathscr{T}\left(S_{g}\right) / \operatorname{Mod}_{0}\left(H_{g}\right)$ where $\mathscr{T}\left(S_{g}\right)$ denotes the Teichmüller space of all conformal structures on the closed surface $S_{g}$ of genus $g$ (up to isotopy) and $\operatorname{Mod}_{0}\left(H_{g}\right)$ denotes the group of (isotopy classes of) homeomorphisms of $S_{g}$ which extend to homeomorphisms of $H_{g}$ which are homotopic to the identity.

We will consider two Schottky groups $\rho_{1}: F_{g} \rightarrow \operatorname{PSL}_{2}(\mathbf{C})$ and $\rho_{2}: F_{g} \rightarrow$ $\mathrm{PSL}_{2}(\mathbf{C})$ to be equivalent if they are conjugate representations. We define Schottky space $\mathscr{S}_{g}$ to be the space of all (equivalence classes of) Schottky groups. It is a theorem of Bers [3], Maskit [24], and Kra [23] that

$$
\mathscr{S}_{g} \cong \mathscr{T}\left(S_{g}\right) / \operatorname{Mod}_{0}\left(H_{g}\right) \text {. }
$$

We define $A H\left(H_{g}\right)$ to be

$$
D\left(F_{g}, \operatorname{PSL}_{2}(\mathbf{C})\right) / \operatorname{PSL}_{2}(\mathbf{C})
$$

where $D\left(F_{g}, \mathrm{PSL}_{2}(\mathrm{C})\right)$ denotes the space of discrete faithful representations of $F_{g}$ into $\mathrm{PSL}_{2}(\mathbf{C})$ and the action of $\mathrm{PSL}_{2}(\mathbf{C})$ is by conjugation. We recall that Schottky space $\mathscr{S}_{g}$ is an open subset of $A H\left(H_{g}\right)$ (Bers [3]) and that $A H\left(H_{g}\right)$ is a closed subset of $R\left(F_{g}, \mathrm{PSL}_{2}(\mathbf{C})\right) / \mathrm{PSL}_{2}(\mathbf{C})$ (Jorgensen [19]) where $R\left(F_{g}, \mathrm{PSL}_{2}(\mathrm{C})\right)$ denotes the space of all representations of $F_{g}$ into $\mathrm{PSL}_{2}(\mathrm{C})$. A sequence of Schottky groups is said to converge algebraically if it converges in $A H\left(H_{g}\right)$.

The first really dramatic step in characterizing when sequences of Schottky groups converge algebraically is due to Thurston [38] (see also Morgan-Shalen $[29,30,31])$. Theorem 1.1 is a key step in Thurston's proof of his geometrization theorem. To state his theorem we need to recall the definition of a doubly incompressible collection of curves.

A mutually disjoint, homotopically distinct system of simple, closed curves $X$ in the boundary $\partial M$ of a compact (orientable) 3-manifold $M$ is doubly incompressible if

1. every compressible curve in $\partial M$ intersects $X$ at least three times,

2. there are no essential annuli (in $M$ ) with boundary in $\partial M-X$, and

3. every maximal abelian subgroup of $\pi_{1}(\partial M-X)$ is mapped to a maximal abelian subgroup of $\pi_{1}(M)$. 
(A curve $\gamma$ in $M$ is said to be compressible if it is null-homotopic in $M$ but not in $\partial M$. A proper map $f:(A, \partial A) \rightarrow(M, \partial M)$ of an annulus $A$ into $M$ is said to be essential if $f_{*}: \pi_{1}(A) \rightarrow \pi_{1}(M)$ is an injection and $f$ is not homotopic as a map of pairs to a map $f^{\prime}$ such that $f^{\prime}(A) \subset \partial M$.)

If $X$ is a finite collection of null-homotopic closed curves in a hyperbolic manifold $N, l_{N}\left(X^{*}\right)$ denotes the total length of their geodesic representatives in $N$. We define $A H(M, X, K)$ to be the set of all Kleinian groups $\rho: \pi_{1}(M) \rightarrow$ $\mathrm{PSL}_{2}(\mathbf{C})$ such that $l_{\mathbf{H}^{3} / \Gamma}\left(X^{*}\right) \leq K$.

Theorem 1.1 (Thurston [38]). If $X$ is a system of doubly incompressible curves on $\partial M$, then $A H(M, X, K)$ is compact for all $K$.

We now recall Thurston's compactification of Teichmüller space by the space of projective measured laminations (see Kerckhoff [21], Fathi-LaudenbachPoenaru [13] or Wolf [41]). This compactification will serve to capture the limiting behavior of the conformal structures.

Let $S_{g}$ be a closed surface of genus $g$ together with a fixed hyperbolic metric $\tau_{0}$. A geodesic lamination on $S_{g}$ is a closed subset $L$ of $S_{g}$ which is a disjoint union of simple geodesics. A measured lamination is an invariant (with respect to projection along $L$ ) measure on arcs transverse to some geodesic lamination $L$ with support on $L . M L\left(S_{g}\right)$ is the space of all measured laminations on $S$ and projective lamination space $P L\left(S_{g}\right)$ is $\left(M L\left(S_{g}\right)-\{\phi\}\right) / \mathbf{R}^{+}$. We will identify $P L\left(S_{g}\right)$ with the set of measured laminations which have length 1 . If $S_{g}$ is a closed surface of genus $g, P L\left(S_{g}\right)$ is a sphere of dimension $6 g-$ 7 which may be identified as the boundary of the Teichmüller space of $S_{g}$, i.e. $\mathscr{T}\left(S_{g}\right) \cup P L\left(S_{g}\right) \cong B^{6 g-6}$. This compactification is natural in the sense that the action of the mapping class group $\mathscr{M}\left(S_{g}\right)$ of $S_{g}$ on $\mathscr{T}\left(S_{g}\right)$ extends continuously to an action on $\mathscr{T}\left(S_{g}\right) \cup P L\left(S_{g}\right)$. (The mapping class group $\mathscr{M}\left(S_{g}\right)$ is the group of isotopy classes of homeomorphism of $S_{g}$.)

Masur [26] studied the action of $\operatorname{Mod}_{0}\left(H_{g}\right)$ on $P L\left(S_{g}\right)$. We will say that a projective measured lamination is in $\mathscr{H}$ if its support is a collection of compressible (in $H_{g}$ ) closed curves in $S_{g}$. Masur proved that the limit set $\mathscr{L}$ of the action of $\operatorname{Mod}_{0}\left(H_{g}\right)$ on $P L\left(S_{g}\right)$ is the closure of $\mathscr{H}$, and that $\operatorname{Mod}_{0}\left(H_{g}\right)$ acts properly discontinuously on $\mathscr{Q}\left(H_{g}\right)$, the set of all laminations in $P L\left(S_{g}\right)$ which have nonzero intersection with every lamination in $\mathscr{L} . \mathscr{O}\left(H_{g}\right)$ is called the Masur domain. (Kerckhoff [22] proved that $\mathscr{O}\left(H_{g}\right)$ has full measure in $P L\left(S_{g}\right)$.) Recall that the limit set is defined to be the smallest closed $\operatorname{Mod}_{0}\left(H_{g}\right)$ invariant subset of $P L\left(S_{g}\right)$. It will be useful to note that $\mathscr{L}$ is also the limit set for the action of $\operatorname{Mod}\left(H_{g}\right)$, the group of all (isotopy classes of) homeomorphisms of $S_{g}$ which extend to homeomorphisms of $H_{g}$, and that $\operatorname{Mod}\left(H_{g}\right)$ also acts properly discontinuously on $\mathscr{O}\left(H_{g}\right)$.

We can therefore adjoin $\mathscr{O}\left(H_{g}\right) / \operatorname{Mod}_{0}\left(H_{g}\right)=\mathscr{B}\left(H_{g}\right)$ to $\mathscr{S}_{g}$ as a sort of "boundary," just as we append $D_{\Gamma} / \Gamma$ as the boundary at infinity of a hyperbolic manifold. This "boundary" does not give a compactification of Schottky space!

We can think of membership in the Masur domain as a generalization of double incompressibility:

Proposition 1.2 (Otal [33]). If $\lambda \in \mathscr{O}\left(H_{g}\right)$ and $C$ is a compressible curve, then $i(\lambda, C)>0$, there are no essential annuli with boundary in $\partial M-\lambda$, and every maximal abelian subgroup of $\pi_{1}(\partial M-\lambda)$ is mapped to a maximal abelian subgroup of $\pi_{1}(M)$. 
Since Masur domain laminations are generalizations of doubly incompressible systems of curves, it is natural to conjecture that if the length of a Masur domain lamination is bounded for a sequence of Schottky groups that the sequence converges (up to subsequence). We will now formalize this conjecture in a way which is reminiscent of Thurston's double limit theorem [37].

Given a divergent sequence $\left\{\rho_{i}\right\} \in \mathscr{S}_{g}$ we consider its parameterization as $\rho_{i}=\tau_{i} \in \mathscr{T}\left(S_{g}\right) / \operatorname{Mod}_{0}\left(H_{g}\right)$. We say that $\left\{\rho_{i}\right\}$ converges into the Masur domain if $\left\{\tau_{i}\right\}$ converges into $\mathscr{B}\left(H_{g}\right)$. In more basic language, a sequence of Schottky groups $\left\{\rho_{i}\right\}$ converges into the Masur domain if and only if there exists a sequence of conformal structures $\left\{\tilde{\tau}_{i}\right\} \in \mathscr{T}\left(S_{g}\right)$ (consistent with $\left\{\tau_{i}\right\}$ ) converging to $\mu \in \mathscr{O}\left(H_{g}\right)$ in Thurston's compactification of Teichmüller space by $P L\left(S_{g}\right)$. When we say $\tilde{\tau}_{i}$ is consistent with $\tau_{i}$ we mean that $\tilde{\tau}_{i}$ is a lift of $\tau_{i} \in \mathscr{T}\left(S_{g}\right) / \operatorname{Mod}_{0}\left(H_{g}\right)$ to $\mathscr{T}\left(S_{g}\right)$.

Conjecture 1.3 (Thurston). If a sequence $\left\{\rho_{i}\right\}$ in $\mathscr{S}_{g}$ converges into the Masur domain, then $\left\{\rho_{i}\right\}$ has a convergent subsequence in $A H(M)$.

Remark. This conjecture is a particular case of a very general conjecture about the algebraic convergence of sequences of convex cocompact Kleinian groups (see [11]).

In this paper we obtain a partial answer to Conjecture 1.3 , unfortunately we will still need to assume some internal information about lengths of curves in the 3-manifold.

Any handlebody has many representations as $S \times I$ where $S$ is a surface with boundary. For example, the handlebody of genus two can be represented in various ways as the punctured torus $\times I$ and the thrice punctured sphere $\times I$. $l_{N_{i}}\left((\partial S)^{*}\right)$ will denote the lengths of the geodesic representatives in $N_{i}$ of the collection of curves which make up the boundary of $S$.

Main Theorem. Let $\left\{\rho_{i}\right\}$ be a sequence of Schottky groups converging into the Masur domain. If $H_{g}=S \times I$ and $l_{N_{i}}\left((\partial S)^{*}\right) \leq K$ (where $N_{i}=\mathbf{H}^{3} / \rho_{i}\left(F_{g}\right)$ ) for all $i$ and some $K$, then $\left\{\rho_{i}\right\}$ has a convergent subsequence in $A H\left(H_{g}\right)$.

The basic logic of the argument is the same as in the proof of the double limit theorem. Consider a sequence $\left\{\tau_{i}\right\}$ in $\mathscr{T}\left(S_{g}\right)$ consistent with $\left\{\rho_{i}\right\}$ and converging to $\mu \in \mathscr{O}\left(H_{g}\right)$. We know that there exists a sequence of measured laminations $\mu_{i}$ (of unit length in $\tau_{0}$ ) converging to $\mu$ such that $l_{\tau_{i}}\left(\mu_{i}\right)$ converges to zero. We then see, in $\S \S 2$ and 3 , that the length of $\mu_{i}$ in $N_{i}$ converges to zero also, and explain what that means.

In $\S 4$ we analyze the structure of the laminations within the manifold; each Masur domain lamination is associated to a current in the handlebody, and thus on $S$. We then show that any Masur domain current binds $S$, in the sense that for all $K$ the set of surfaces in $\mathscr{T}(S)$ on which this current has length less than $K$ is precompact in the Fricke space of all hyperbolic structures on $S$.

In $\S 5$, we show that currents in $N$ (which do not "wind" too much about components of $\partial S$ ) may be represented by currents of similar length on a pleated surface whose pleating locus' only compact leaves are components of $\partial S$. Within each of the hyperbolic manifolds associated to our sequence of Schottky groups we can form such a pleated surface, and the above analysis shows that the hyperbolic structures on these pleated surfaces converges. Thus our sequence of representations converges algebraically. 
In $\S 6$, we explicitly assemble the proof and state an internal version of our main theorem which follows from our technique of proof. We will also discuss, briefly, the tree-theoretic approach to this theorem.

I would like to thank Sergio Fenley, Curt McMullen, Yair Minsky, JeanPierre Otal, and Bill Thurston for their help at various stages of this project. I would like to thank Howard Masur and the referee for their comments on the original version of this paper. The results in this paper formed a portion of my $\mathrm{Ph}$.D. thesis completed at Princeton University under the supervision of Bill Thurston.

\section{Realizations of Masur domain laminations}

In this section we will review some of Otal's [33] important work on realizations of Masur domain laminations within hyperbolic 3-manifolds. By a realization of a lamination $\lambda$ we will mean a pleated surface $p: S_{g} \rightarrow N$ such that $p(\lambda)$ is a collection of geodesics and $p$ is homotopic to the inclusion $i: \partial H_{g} \rightarrow H_{g}$. We recall the definition of pleated surfaces (see [39], [36] and/or [12] for a detailed discussion of pleated surfaces). Pleated surfaces are a powerful tool for understanding the image of a lamination in a hyperbolic 3-manifold.

Definition 2.1. A pleated surface in a hyperbolic 3-manifold $N$ is a surface $S$ with a hyperbolic metric $\tau$ of finite area (such that each component of $\partial S$ is a geodesic), together with a map $p:(S, \tau) \rightarrow N$ which is an isometry with respect to the metric $\tau$ on $S$ such that every $x \in S$ is in the interior of some geodesic arc which is mapped by $p$ to a geodesic arc in $N .{ }^{1}$

Also, $p$ must take any cusp of $S$ to a cusp of $N$. A point $x \in S$ is in the pleating locus if it is in the interior of only one geodesic arc which is mapped to a geodesic arc.

We recall that the pleating locus is a geodesic lamination which is mapped to a union of geodesics in $N$ and that the complement of the pleating locus is mapped totally geodesically. Otal demonstrated that every Masur domain lamination has a realization by a pleated surface.

Proposition 2.2 (Otal [33]). Let $\Gamma$ be a Schottky group, and $h$ a fixed identification of $\left(\mathbf{H}^{3} \cup D_{\Gamma}\right) / \Gamma$ with $H_{g}$. If $\lambda \in \mathscr{O}\left(H_{g}\right)$ and $N=\mathbf{H}^{3} / \Gamma$, then there exists a pleated surface $p:\left(S_{g}, \tau\right) \rightarrow N$ homotopic to the inclusion $i: \partial H_{g} \rightarrow H_{g}$ which maps $\lambda$ to a collection of geodesics in $N$.

If $\lambda$ is a geodesic lamination which a pleated surface takes to a collection of geodesics there is an associated projective map $P: \lambda \rightarrow \mathbf{P}(N)$ (where $\mathbf{P}(N)$ is the projective tangent bundle of $N$ ) which takes a point $x$ on a leaf $l$ of $\lambda$ to the vector in $\mathbf{P}(N)$ lying above $x$ and in the direction of $l$.

Proposition 2.3 (Otal [33]). If $p:\left(S_{g}, \tau\right) \rightarrow N$ is a pleated surface and $\lambda$ is a geodesic lamination which is mapped to a collection of geodesics in $N$ and contains the support of a Masur domain lamination, then $P$ is a homeomorphism onto its image.

\footnotetext{
${ }^{1}$ By an isometric map we mean a map which takes rectifiable arcs in $S$ to rectifiable arcs of the same length in $N$.
} 
We want to think of the image of a Masur domain lamination as a current in $N$ so we must recall the definition of a current (see Bonahon [7, 8, 9] for a complete discussion of currents).

The convex core of a hyperbolic 3-manifold is the smallest convex submanifold such that the inclusion map is a homotopy equivalence. $N$ is said to be convex cocompact if its convex core is compact. Notice that if $\Gamma$ is a Schottky group, its convex core is a handlebody of genus $g$ and hence $N=\mathbf{H}^{3} / \Gamma$ is convex cocompact.

A (geodesic) current on a hyperbolic manifold $N$ is a (positive) transverse invariant measure on the geodesic flow of $N$ whose support is contained within the projective tangent bundle of the convex core. (Equivalently, if $N=\mathbf{H}^{n} / \Gamma$, we may think of a current as a $\Gamma$-invariant measure on $L_{\Gamma} \times L_{\Gamma}-\Delta$.) We denote the space of currents on $N$ by $\mathscr{C}(N)$. If the support of a current $c$ is a closed geodesic we define the length of $c, l_{N}(c)$, to be the length of that closed geodesic times the transverse measure of $c . l_{N}$ extends to a linear map $l_{N}: \mathscr{C}(N) \rightarrow \mathbf{R}_{+} \cup\{0\}$ which is continuous if $N$ is convex cocompact [9].

If $N$ is a hyperbolic surface and $\alpha$ and $\beta$ are two closed geodesics on $N$ which intersect transversely we define their geometric intersection number $i(\alpha, \beta)$ to be the number of points in $\alpha \cap \beta$. $i$ extends to a symmetric, bilinear map

$$
i: \mathscr{C}(N) \times \mathscr{C}(N) \rightarrow \mathbf{R}_{+} \cup\{0\}
$$

which is again continuous if $N$ is convex cocompact [7]. The measured laminations on $N$ may be identified with the currents $c$ such that $i(c, c)=0$.

If $N=\mathbf{H}^{s} / \Gamma$ and $N^{\prime}=\mathbf{H}^{t} / \Gamma^{\prime}$ are homotopy equivalent convex cocompact hyperbolic manifolds, then this homotopy equivalence induces a homeomorphism $h: L_{\Gamma} \rightarrow L_{\Gamma^{\prime}}$ (see Floyd [14]) and hence an identification of $\mathscr{C}(N)$ with $\mathscr{C}\left(N^{\prime}\right)$, in this way it is reasonable to speak of the same current in homotopy equivalent hyperbolic manifolds. If $S=\mathbf{H}^{2} / \Gamma$ and $S^{\prime}=\mathbf{H}^{2} / \Gamma^{\prime}$ are two homeomorphic convex cocompact hyperbolic surfaces then the identification of $\mathscr{C}(S)$ to $\mathscr{C}\left(S^{\prime}\right)$ preserves intersection number but not length. We can thus think of $\mathscr{C}\left(S_{g}\right)$ and $M L\left(S_{g}\right)$ as being topological objects with well-defined intersection number independent of the hyperbolic structure on $S_{g}$.

In particular, if $\Gamma$ is a Schottky group and we are given a homotopy equivalence of $N=\mathbf{H}^{3} / \Gamma$ with a convex cocompact hyperbolic surface $S_{0}$, then $\mathscr{C}(N)$ is identified with $\mathscr{C}\left(S_{0}\right)$. This identification will be central to our proof, as we will identify $H_{g}$ with $S \times I$ where $S$ is a compact surface.

Let $\Gamma$ be a Schottky group and $N=\mathbf{H}^{3} / \Gamma$. Every lamination $\lambda \in \mathscr{O}\left(H_{g}\right)$ which has as support a collection of closed geodesics, is associated by its pleated surface realization to a collection of geodesics in $N$. We may now think of this set of geodesics as a current on $N$, where we give each geodesic the same measure as the associated component of $\lambda$; denote this current $F(\lambda)$. Otal has shown that $F$ extends to a map $F: \mathscr{O}\left(H_{g}\right) \rightarrow \mathscr{C}(N)$ where $F(\lambda)$ is a current with support $p(\lambda)$ where $\lambda$ is mapped to a collection of geodesics in $N$ by the pleated surface $p:\left(S_{g}, \tau\right) \rightarrow N$. (Recall that we represent $\mu \in \mathscr{O}\left(H_{g}\right)$ by the unit length measured lamination in its class.) The support of this current is the image of $\lambda$ by a pleated surface which maps $\lambda$ isometrically. If $\lambda=h\left(\lambda^{\prime}\right)$ where $h$ is the homeomorphism of $P L\left(S_{g}\right)$ induced by an element of $\operatorname{Mod}_{0}\left(H_{g}\right)$ then $F(\lambda)=F\left(\lambda^{\prime}\right)$ so $F$ descends to a map $F: \mathscr{B}\left(H_{g}\right) \rightarrow \mathscr{C}(N)$. 
Theorem $2.4\left(\right.$ Otal [33]). $F: \mathscr{B}\left(H_{g}\right) \rightarrow \mathscr{C}(N)$ is a homeomorphism of $\mathscr{B}\left(H_{g}\right)$ onto its image in $\mathscr{C}(N)$.

Remark. In the proof of our main theorem it is only necessary to use the fact that $F$ is injective and continuous.

\section{The PoINCARÉ METRIC}

In this section we will convert information about the limiting behavior of the conformal structures to usable information about the internal geometry of the associated sequence of hyperbolic manifolds. We will first introduce the proposition which will allow us to make this conversion.

Proposition 3.1 [10]. Given $A>0$, there exists $K$ such that if $\Gamma$ is a finitely generated Kleinian group such that every compressible curve on $R=D(\Gamma) / \Gamma$ has length (in the Poincare metric on $R$ ) greater than $A$, and $\gamma$ is a curve on $R$, then $l_{N}\left(\gamma^{*}\right) \leq K l_{R}(\gamma)$ where $N=\mathbf{H}^{3} / \Gamma$.

The following corollary follows directly from Proposition 3.1, since length is continuous in both $M L\left(S_{g}\right)$ and $\mathscr{C}(N)$. (Notice that if $\lambda$ is a measured lamination whose projective class lies in the Masur domain, then $\lambda=k \mu$ where $\mu$ is a unit length representative of $\lambda$ 's projective class, so we may define $F(\lambda)=$ $k F(\mu)$.)

Corollary 3.2. If $\Gamma$ is a Schottky group such that every compressible curve in $R=D_{\Gamma} / \Gamma$ has length (in the Poincare metric on $R$ ) greater than $A$ and $\lambda \in$ $M L\left(S_{g}\right)$ is a measured lamination whose projective class lies in $\mathscr{O}\left(H_{g}\right)$, then

$$
l_{N}(F(\lambda)) \leq K l_{R}(\lambda)
$$

where $N=\mathbf{H}^{3} / \Gamma$ and $K$ is as in Proposition 3.1.

The limiting behavior of the conformal geometry is captured by the following property of Thurston's compactification:

Theorem 3.3 (Thurston). Let $S_{g}$ be a closed surface of genus $g$, and $\tau_{n} \in$ $\mathscr{T}\left(S_{g}\right)$ a sequence of hyperbolic structures converging to $\mu \in P L\left(S_{g}\right)$ in the Thurston compactification of $\mathscr{T}\left(S_{g}\right)$. Then there exists a sequence of measured laminations $\mu_{n} \in M L\left(S_{g}\right)$ and $K>0$, such that for any current $c \in \mathscr{C}\left(S_{g}\right)$

$$
i\left(\mu_{n}, c\right)+K l_{\tau_{0}}(c) \geq l_{\tau_{n}}(c) \geq i\left(\mu_{n}, c\right) .
$$

Also, $l_{\tau_{n}}\left(\mu_{n}\right)$ remains bounded and the sequence of projective classes $\left\{\left[\mu_{n}\right]\right\}$ converges to $\mu$.

Remark. Thurston has never written down a proof of this property although it can be proven using the techniques of [40]. This theorem is essentially proven in Lemma II. 1 in exposé 8 in Fathi-Laudenbach-Poenaru [13], it only remains to notice that the proof applies equally well to all closed curves and to identify the combinatorial constant obtained as $K l_{\tau_{0}}(c)$. The theorem may also be proved using the techniques of Wolf [41]. In fact we only need to use the fact that $l_{\tau_{n}}\left(\mu_{n}\right) / l_{\tau_{0}}\left(\mu_{n}\right)$ goes to zero and that $l_{\tau_{n}}(c) \geq i\left(\mu_{n}, c\right)$ for all $c \in \mathscr{C}(S)$. These facts are easier to prove than the whole theorem and can be obtained as in Lemma 4.6 and Corollary 4.5(c) in [41].

In order to use Corollary 3.2 we need the following lemma. 
Lemma 3.4. Let $\left\{\rho_{j}\right\}$ be a sequence in $\mathscr{S}_{g}$ converging into the Masur domain and $A>0$ some constant. There exists a $J$ such that if $j \geq J, \delta_{j} \in \mathscr{T}\left(S_{g}\right)$ is consistent with $\rho_{j}$, and $\gamma$ is any compressible curve in $S_{g}$,

$$
l_{\delta_{j}}(\gamma) \geq A
$$

Proof of 3.4. Let $\left\{\tau_{j}\right\} \in \mathscr{T}\left(S_{g}\right)$ be a sequence of conformal structures on $S_{g}$ consistent with $\left\{\rho_{j}\right\}$ and converging to $\mu \in \mathscr{O}\left(H_{g}\right)$ and let $\left\{\mu_{j}\right\}$ be the sequence given by Theorem 3.3. Suppose there exist compressible curves $m_{j}$ with length less than $A$ for arbitrarily large $j$. This would imply, by Theorem 3.3, that $i\left(m_{j}, \mu_{j}\right) \leq A$. Then $\left\{m_{j} / l_{\tau_{0}}\left(m_{j}\right)\right\}$ converges (up to subsequence) to $m \in \mathscr{L} \subset M L\left(S_{g}\right)$ such that $i(m, \mu)=0$ which contradicts $\mu$ 's presence in the Masur domain. This implies that there exists some $J$ such that if $j \geq J$, any compressible curve has length greater than $A$ in $\tau_{j}$. But since the set of compressible curves is invariant by any element of $\operatorname{Mod}_{0}\left(H_{g}\right)$ and $\delta_{j}=h\left(\tau_{j}\right)$ for some $h \in \operatorname{Mod}_{0}\left(H_{g}\right)$ the lemma follows.

We now combine all the above analysis to obtain the precise statement which we use in our proof.

Corollary 3.5. If $\left\{\rho_{i}: F_{g} \rightarrow\right.$ Isom $\left._{+}\left(\mathbf{H}^{3}\right)\right\}$ is a sequence of Schottky groups converging to $\mu \in \mathscr{B}\left(H_{g}\right)$, then there exists a sequence of currents $c_{i}=F\left(\mu_{i}\right)$ converging to $F(\mu)$, such that $l_{N_{i}}\left(c_{i}\right)$ goes to zero (where $N_{i}=\mathbf{H}^{3} / \rho_{i}\left(F_{g}\right)$ ).

Proof of 3.5. Let $\left\{\tau_{i}\right\}$ be a sequence of conformal structures on $S_{g}$ consistent with $\left\{\rho_{i}\right\}$ and converging to $\widetilde{\mu} \in \mathscr{O}\left(H_{g}\right)$ (where $\widetilde{\mu}$ is a lift of $\mu \in \mathscr{B}\left(H_{g}\right)$ to $\left.\mathscr{O}\left(H_{g}\right)\right)$. Let $\mu_{i}$ be as in Theorem 3.3, $\mu_{i}^{\prime}=\mu_{i} / l_{\tau_{0}}\left(\mu_{i}\right)$ and $c_{i}=F\left(\mu_{i}^{\prime}\right)$. Notice that since the Masur domain is an open set, there exists some $I$ such that for all $i \geq I, \mu_{i}$ is in the Masur domain. When $i<I$ we may choose $\mu_{i}$ to be any Masur domain lamination.

Then by Proposition 3.1 and Lemma 3.4, there exists $K>0$ such that for large enough $i$

But we also know that $l_{\tau_{i}}\left(\mu_{i}^{\prime}\right)$ converges to 0 .

$$
l_{N_{i}}\left(c_{i}\right) \leq K l_{\tau_{i}}\left(\mu_{i}^{\prime}\right)
$$

\section{MASUR DOMAIN CURRENTS BIND}

We now suppose that we have a fixed homeomorphism of $H_{g}$ to $S \times I$ where $S$ is a compact surface with boundary. In particular, this induces a fixed isomorphism of $\pi_{1}\left(H_{g}\right)$ and $\pi_{1}(S)$. So given a Schottky group $\rho: \pi_{1}\left(H_{g}\right) \rightarrow \mathrm{PSL}_{2}(\mathbf{C})$, this gives a well-defined (up to homotopy) homotopy equivalence of $N=\mathbf{H}^{3} / \Gamma$ with $S$. If we give the interior $S_{0}$ of $S$ a convex cocompact hyperbolic structure $\tau_{0}$ then this induces an identification of $\mathscr{C}(N)$ with $\mathscr{C}\left(S_{0}\right)$. The convex core of $S_{0}$ is homeomorphic to $S$ and all our currents live on the convex core, so we will identify $\mathscr{C}\left(S_{0}\right)$ with $\mathscr{C}(S)$. So we will think of our original map $F: \mathscr{B}\left(H_{g}\right) \rightarrow \mathscr{C}(N)$, as a map $F: \mathscr{B}\left(H_{g}\right) \rightarrow \mathscr{C}(S)$.

In this section we will undertake an analysis of the currents on $S$ which arise as images, by the map $F$, of Masur domain laminations. We will call such currents Masur domain currents. Our aim is to prove that Masur domain currents "bind" $S$.

4.1 The topology of Masur domain currents. In this section we prove that Masur domain currents intersect every measured lamination on $S$ which does 
not consist entirely of components of $\partial S$. Results in this section overlap with results of Floyd [15] and Otal [33].

Let $M L_{T}(S)$ denote the set of measured laminations on $S$ such that all halfleaves either do not intersect the boundary or intersect the boundary (which is a collection of geodesics) perpendicularly. Let $M L_{T}^{0}(S)$ denote the set of laminations in $M L_{T}(S)$ which do not contain any component of $\partial S$ as a leaf. Notice that $P L_{T}(S)$ and $P L_{T}^{0}(S)$ are both compact, where we may think of $P L_{T}(S)$ as the set of laminations in $M L_{T}(S)$ which have unit length. (Notice that we could think of measured laminations in $M L_{T}(S)$ as the restrictions of measured laminations on $S_{0}$. Alternatively we can identify $M L_{T}(S)$ with the set of measured laminations on $D S$, the double of $S$, which are invariant with respect to the obvious involution.)

Theorem 4.1. A Masur domain current intersects every lamination in $M L_{T}^{0}(S)$.

Proof of 4.1. Suppose that $c=F(\mu)$ does not intersect a homotopically nontrivial simple closed curve $\gamma$ on $S$. Then if $f_{\gamma}$ denotes the Dehn twist (of $S$ ) about $\gamma, f_{\gamma}^{*}(c)=c$. Therefore if $h$ denotes the twist about the annulus $\gamma \times I$ in $S \times I, F(h(\mu))=F(\mu)$ which contradicts Theorem 2.4.

Now suppose that $i(c, \lambda)=0$ for some minimal lamination $\lambda$, either there exists $\varepsilon>0$ such that the support of $c$ does not intersect the $\varepsilon$-neighborhood of $\lambda$ or $\lambda$ is a component of $c$. In the first case either the $\varepsilon$-neighborhood of $\lambda$ contains a homotopically trivial closed curve (in which case $c$ misses that curve) or the support of $\lambda$ contains a bi-infinite geodesic $l$ each of whose ends intersects the convex core perpendicularly. We may then form a homotopically nontrivial curve $\gamma$ which $c$ does not intersect by appending arcs in the boundary of the convex core to the intersection of $l$ with the convex core. Thus, as above, $c$ is fixed by the Dehn twist about $\gamma$ and $\mu$ cannot lie in the Masur domain.

Now suppose that $\lambda$ is a component of $c$. If $\lambda$ is not filling, i.e. if one of its complementary regions is not either simply connected or a half-open annulus including $\partial S$, then the boundary of the $\varepsilon$-neighborhood contains a homotopically nontrivial simple closed curve which is not homotopic to $\partial S$ and which $c$ must not intersect. Therefore $\lambda$ must be filling, and thus $\lambda$ must be equal to $c$.

If $\mu$ projects to a lamination $\lambda$ on $S$ which is not a simple closed geodesic, we then show that we may approximate $\mu$ by laminations whose projections are simple closed geodesics on $S$. Since none of these laminations lies in the Masur domain (by the argument given in the first paragraph of the proof), $\mu$ itself cannot lie in the Masur domain. Recall that this lamination has support contained entirely in the interior of the convex core. We will present two, essentially equivalent, ways to construct this approximation.

Here is the first technique: form two pleated surfaces both of which contain $F(\mu)$ in their image one with base surface $S$, denoted $p:(S, \tau) \rightarrow N$, wahich maps $\lambda$ to a collection of geodesics in $N$ and the other with base surface $S_{g}$, denoted $p^{\prime}:\left(S_{g}, \tau^{\prime}\right) \rightarrow N$, which maps $\mu$ to the same collection of geodesics in $N$. One may construct an approximation of $F(\mu)$ by a sequence of simple closed curves $\gamma_{i}$ (on $p(S)$ ) obtained by running along a boundary leaf of $F(\mu)$ for a long time and then joining the two ends by a short arc (see [12], for example). We now wish to realize this approximation (on $S$ ) by a simple closed curve on $\partial H_{g}$ which projects to the same geodesic in $N$, i.e. we wish to 
construct embeddings $r_{i}: \gamma_{i} \rightarrow S_{g}$ in such a way that $r_{i}\left(\gamma_{i}\right)$ is near to $\mu$ and so that $p^{\prime} \circ r_{i}\left(\gamma_{i}\right)$ is homotopic to $\gamma_{i}$. First, $r_{i}$ maps the portion of $\gamma_{i}$ which lies on $F(\mu)$ to the portion of $\mu$ identified with it by $p^{\prime}$. We then only need to decide where the short arc is mapped. The short arc (on $p(S))$ is made up of a (at most) countable number of short intervals in the complement of $F(\mu)$ and a closed set which lies on $F(\mu)$, again we identify the part which lies on $F(\mu)$ pointwise with its pre-image (on $\mu$ ) under $p^{\prime}$. So we need only say how to map in an interval running from a boundary leaf to a boundary leaf. Any boundary leaf in $F(\mu)$ is the image of a boundary leaf of $\mu$ and two leaves of $F(\mu)$ are asymptotic if and only if the associated leaves in $\mu$ are. However, the pleating locus of $p^{\prime}$ may contain some numbers of isolated leaves which lie between the two associated boundary leaves on $S_{g}$. However, if our arc was chosen short enough we are in a neighborhood of the "spike" of the two boundary leaves which looks simply like the identification of some finite number of "spikes" of ideal triangles. Thus we may simply move across along horocycles in the spike. The result clearly has the desired properties.

A second, perhaps simpler, way to look at this same proof is via train tracks in 3-manifolds. Consider the intersection of a small neighborhood $U$ of $F(\mu)$ in $N$ with a small neighborhood of $p^{\prime}(\mu)$ in $p^{\prime}\left(\partial H_{g}\right)$ and the intersection of $U$ with a small neighborhood of $p(\lambda)$ in $p(S)$. These intersections give train-tracks in $\partial H_{g}$ and $S$, respectively, which carry laminations which define the same currents in $N$. (See Bonahon [7] for a discussion of train tracks.) But the train track on $S$ carries simple closed curves arbitrarily close to $\lambda$, thus there exist laminations (on $\partial H_{g}$ ) arbitrarily near to $\mu$ which project to currents associated to simple closed curves on $S$.

To complete the proof of our main theorem we will need the following lemma which assures us that no component of a Masur domain lamination projects to a component of $\partial S$.

Lemma 4.2. No component of a Masur domain lamination is homotopic (in $\mathrm{H}_{\mathrm{g}}$ ) to a component of $\partial S$.

Proof of 4.2. Let $\gamma$ be a component of a Masur domain lamination $\mu$. Suppose $\gamma$ is homotopic to a component of $\partial S$.

If $S$ has more than one component then $\gamma$ is homotopic to a generator of $\pi_{1}\left(H_{g}\right)$. Therefore, if we attach a 2-handle $D_{\gamma}$ to $H_{g}$ along $\gamma$, the resulting manifold $H_{g} \cup D_{\gamma}$ has as fundamental group the free group on $g-1$ generators. Hence, $H_{g} \cup D_{\gamma}$ is a handlebody of genus $g-1$. Therefore, see Jaco [18], there is a compressible curve $\beta$ on $\partial H_{g}$ which is disjoint from $\gamma$. Notice that we may assume that $\beta$ is nonseparating, because if $\beta$ separated $S_{g}$, then $\beta$ would bound a disk $D$ in $H_{g}$ and $\beta \cup D$ would separate $H_{g}$. In which case, the component of $H_{g}-(\beta \cup D)$ which does not contain $\gamma$ is a handlebody of lower genus, and would itself contain a compressible curve which was nonseparating on $S_{g}$. Moreover, $\gamma$ itself must be nonseparating. We may then choose an embedded arc $k$ with its endpoints on $\beta$ which intersects $\gamma$ once and $\beta$ only at its endpoints and approaches $\beta$ from opposite sides. Let $C$ be the compressible curve constructed by running around $\beta$ across $k$, around $\beta$ again and backwards across $k$. Notice that $C$ is compressible and is homotopic to a simple closed geodesic $\alpha$. Then if $D_{\gamma}^{n}(\alpha)$ is the curve obtained by Dehn twisting $\alpha n$ times about $\gamma, D_{\gamma}^{n}(\alpha)$ is compressible (for all $n$ ) and $\left\{D_{\gamma}^{n}(\alpha) / l_{\tau}\left(D_{\gamma}^{n}(\alpha)\right)\right\}$ 
converges to $\gamma / l_{\tau}(\gamma)$, so $\gamma$ is a limit of a sequence of compressible curves and thus is contained in $\mathscr{L}$. Therefore, by definition (since $i(\mu, \gamma)=0), \mu$ cannot lie in the Masur domain.

Now suppose that $S$ has only one boundary component. We may again attach a 2-handle $D_{\gamma}$ to $H_{g}$ along $\gamma . \pi_{1}\left(H_{g} \cup D_{\gamma}\right)$ is isomorphic to the fundamental group of a closed surface $T_{g / 2}$ of genus $g / 2$, and thus, by basic 3-manifold theory (see Hempel [17]), $H_{g} \cup D_{\gamma}$ is homeomorphic to $T_{g / 2} \times I$. Thus there is a homeomorphism of $H_{g}$ to $S \times I$ where $\gamma$ is homotopic to $\partial S \times\left\{\frac{1}{2}\right\}$ (see Gordon [16]). $\gamma$ is thus disjoint from the boundary of some essential annulus $A$ (obtained as $\beta \times I$ for some curve $\beta$ in $S$ ) and is therefore fixed by the twist about the annulus $A$ and hence does not itself lie in the Masur domain. If $\lambda$ is any other component of $\mu$ it may be approximated by nonseparating simple closed curves $\gamma_{i}$ each of which misses a nonseparating compressible curve, and thus, as above, lies in $\mathscr{L}$. (Notice that each of these nonseparating curves $\gamma_{i}$ projects to a simple closed curve on $S$ which is homotopic to a generator of $\pi_{1}(S)$, so we also could have proceeded as in the first paragraph of the proof to show that $\gamma_{i}$ lies in $\mathscr{L}$.) Therefore, $\lambda$ lies in $\mathscr{L}$ and $\mu$ itself cannot lie in the Masur domain.

4.2 Binding currents. We first recall that the Fricke space $\widehat{\mathscr{T}}(S)$ of a surface $S$ with boundary is the closure of the embedding of $\mathscr{T}(S)$ into

$$
D\left(\pi_{1}(S), \mathrm{PSL}_{2}(\mathbf{R})\right) / \mathrm{PSL}_{2}(\mathbf{R})
$$

(see Bers-Gardiner [4]). $\widehat{\mathscr{T}}(S)$ consists of the Teichmüller space $\mathscr{T}(S)$ of all hyperbolic structures on the surface with geodesic boundary union the Teichmüller spaces of all the surfaces which arise by pinching components of the boundary. (Notice that there is a one-to-one correspondence between the set of hyperbolic structures on $S$ with geodesic boundary and the set of complete hyperbolic structures on $S_{0}$.) In the Fenchel-Nielsen coordinates (see Abikoff [1]), if $S$ is a surface of genus $g$ with $b$ geodesic boundary components (this is sometimes called a surface of type $(g, 0, b))$,

$$
\mathscr{T}(S)=\mathbf{R}_{+}^{b} \times \mathbf{R}_{+}^{b} \times \mathbf{R}_{+}^{3 g-3} \times \mathbf{R}^{b} \times \mathbf{R}^{3 g-3}
$$

where the first $b$ coordinates are the lengths of the boundary geodesics, the next $b+3 g-3$ coordinates are lengths of internal curves, and the last $b+3 g-3$ coordinates measure twist. In these same coordinates,

$$
\widehat{\mathscr{T}}(S)=\left(\mathbf{R}_{+} \cup\{0\}\right)^{b} \times \mathbf{R}_{+}^{b} \times \mathbf{R}_{+}^{3 g-3} \times \mathbf{R}^{b} \times \mathbf{R}^{3 g-3} .
$$

We will need to make use of the following theorem, which is a simple generalization of Thurston's compactification theorem for the Teichmüller space of a closed surface.

Theorem 4.3. Let $S$ be a compact hyperbolic surface with geodesic boundary (i.e. of type $(g, 0, b))$, and $\tau_{n} \in \mathscr{T}(S)$ a sequence of hyperbolic metrics leaving all compact subsets of $\mathscr{T}(S)$. Then there exists a subsequence $\left\{n_{j}\right\}$ and a sequence of measured laminations $\mu_{n_{j}} \in M L_{T}(S)$ and $K>0$, such that for any current $c$

$$
i\left(\mu_{n_{j}}, c\right)+K l_{\tau_{0}}(c) \geq l_{\tau_{n_{j}}}(c) \geq i\left(\mu_{n_{j}}, c\right) .
$$

Also, $l_{\tau_{n_{j}}}\left(\mu_{n_{j}}\right)$ remains bounded. 
Remark. Thurston does not explicitly deal with the case of surfaces with geodesic boundary, but we may obtain the result by considering $D S$, the double of $S$, which is formed by attaching two copies of $S$ at their boundary. If we have a sequence of hyperbolic structures $\tau_{n}$ on $S$, they induce, by doubling, a sequence of hyperbolic structures $D \tau_{n}$ on $D S$. If we follow Fathi-LaudenbachPoenaru's proof of Theorem 3.3 we need only make sure that at each stage the pair of pants decomposition $\mathscr{K}$ is invariant with respect to the obvious involution. In Wolf's approach one need only choose the base metric $\sigma$ on $D S$ to be invariant with respect to the obvious involution of $D S$, then the sequence $\left\{\mu_{n}\right\}$ obtained in Theorem 3.3 will also be invariant with respect to the involution. This argument yields the above result.

We will say that a current binds $S$ if given $K>0$ the subset of the Teichmüller space $\mathscr{T}(S)$ on which the length of $c$ is $\leq K$ is precompact in $\widehat{\mathscr{T}}(S)$. One case of binding is the case used in [37], where Thurston proves that the union of two laminations which cut up a closed surface into certain simple pieces binds. One can generalize the discussion in [37] to prove

Proposition 4.4. A current $c$ on a surface $S$ binds if and only if $i(\lambda, c)>0$ for all $\lambda \in M L_{T}^{0}\left(S_{0}\right)$.

The motivation behind all this work was to prove that Masur domain currents bind which is now an easy consequence of Proposition 4.4 and Theorem 4.1.

Corollary 4.5. If $H_{g}=S \times I$, any Masur domain current binds $S$.

Proof of 4.4. It is easy to see that if a current binds it must intersect every measured lamination $\lambda \in M L_{T}^{0}(S)$. If $i(\lambda, c)=0$ we may use $\lambda$ to obtain a divergent sequence of hyperbolic structures in which $l(c)$ remains bounded. This sequence may be obtained by pinching $S$ along $\lambda$ using either stretch maps [40] or harmonic maps [41]. (It is sometimes helpful to think of pinching the doubled surface along $D \lambda$.)

To prove the converse, let $\tau_{n}$ be a sequence in $\mathscr{T}(S)$ such that $l_{\tau_{n}}(c) \leq K$ for all $n$ and $\left\{\tau_{n}\right\}$ does not have a convergent subsequence in $\widehat{\mathscr{T}}(S)$. We then pass to a subsequence (without changing the notation) given by Theorem 4.3. Let $\left\{\mu_{n}\right\}$ be as in Theorem 4.3. Notice that

$$
\mu_{n}=\sum_{\gamma \in \partial S} r_{n}(\gamma)[\gamma]+\mu_{n}^{\prime}
$$

where $\mu_{n}^{\prime} \in M L_{T}^{0}(S)$ and $[\gamma]$ is a unit length (in $\tau_{0}$ ) representative of $\gamma$. There exists a finite collection $\left\{\gamma_{1}, \ldots, \gamma_{k}\right\}$ of curves on $S$, none of which is homotopic to a boundary component, such that $\sum_{i=1}^{k} l_{\tau_{n}}\left(\gamma_{i}\right)$ is bounded if and only if $\rho_{n}$ converges (up to subsequence) in $\widehat{\mathscr{T}}(S)$. (For example, we may take the images of a doubly incompressible system of curves on the handlebody $S \times I$.) By Theorem 4.3, this implies that $i\left(\gamma_{i}, \mu_{n}\right)=i\left(\gamma_{i}, \mu_{n}^{\prime}\right)$ goes to infinity for some $i$, in particular $l_{\tau_{0}}\left(\mu_{n}^{\prime}\right)$ goes to infinity. But the set of laminations in $M L_{T}^{0}(S)$ whose length is 1 is compact so there exists a minimum for $c$ 's intersection number with any such lamination. This implies that $i\left(c, \mu_{n}^{\prime}\right)$, and thus $l_{\tau_{n}}(c)$, goes to infinity, which is a contradiction. 
In the proof of our main theorem we will use a property which is implicit in the technique of proof but not formally contained in the statement of Proposition 4.4.

Proposition 4.6. Let $c$ be a current which binds $S$ and $\left\{c_{n}\right\}$ a sequence of currents converging to $c$, and $\left\{\tau_{n}\right\}$ a sequence of hyperbolic metrics on $S$ such that $l_{\tau_{n}}\left(c_{n}\right) \leq K$. Then $\left\{\tau_{n}\right\}$ converges in $\widehat{\mathscr{T}}(S)$ (up to subsequence) to a hyperbolic metric $\tau$ on $S$ such that $l_{\tau}(c) \leq K$.

\section{EFFICIENCY OF PLEATED SURFACES}

We now obtain a refined version of efficiency of pleated surfaces (see Thurston [37]), which gives us information about currents as well as laminations. Roughly, any current in the 3-manifold (which does not wind too much about any component of $\partial S$ ) has a representative of reasonably similar length on a pleated surface with a finite leaved pleating locus whose only compact leaves are components of $\partial S$.

5.1 Alternation number. We first extend Thurston's definition of the alternation number $a(\lambda, \mu)$ of a measured lamination $\mu$ with a finite-leaved maximal lamination $\lambda$ on $S$, to the situation of currents. The discussion is almost the same as in [37], and if you are familiar with that discussion it is probably unnecessary to read this section.

Let $\lambda$ be a finite-leaved geodesic lamination with support in $S$. We will say that $\lambda$ is maximal if every component of $S-\lambda$ is an ideal triangle. First we define the alternation number of a closed curve $c$ with a finite-leaved maximal lamination $\lambda$. If $c$ is a leaf of $\lambda$ we define $a(\lambda, c)=0$. Otherwise there is a countable set of intersections of $\lambda$ with $c$. If $x$ and $y$ are two consecutive intersections of $\lambda$ with $c$, then $x$ and $y$ lie on two sides of an ideal triangle in $B-\lambda$, so the leaves of $\lambda$ through $x$ and $y$ are asymptotic. If $x, y$, and $z$ are three consecutive intersections of $\lambda$ with $c$, we will say that $y$ is a boundary intersection if the leaves of $\lambda$ through $x$ and $y$ are asymptotic on the opposite side of $c$ from the side on which the leaves of $\lambda$ through $y$ and $z$ are asymptotic. See Figure 1.

If a point $y$ of $\lambda \cap c$ is an accumulation point of intersections, then the leaf of $\lambda$ through $y$ is closed and the leaves of $\lambda$ near $y$ spiral towards this closed
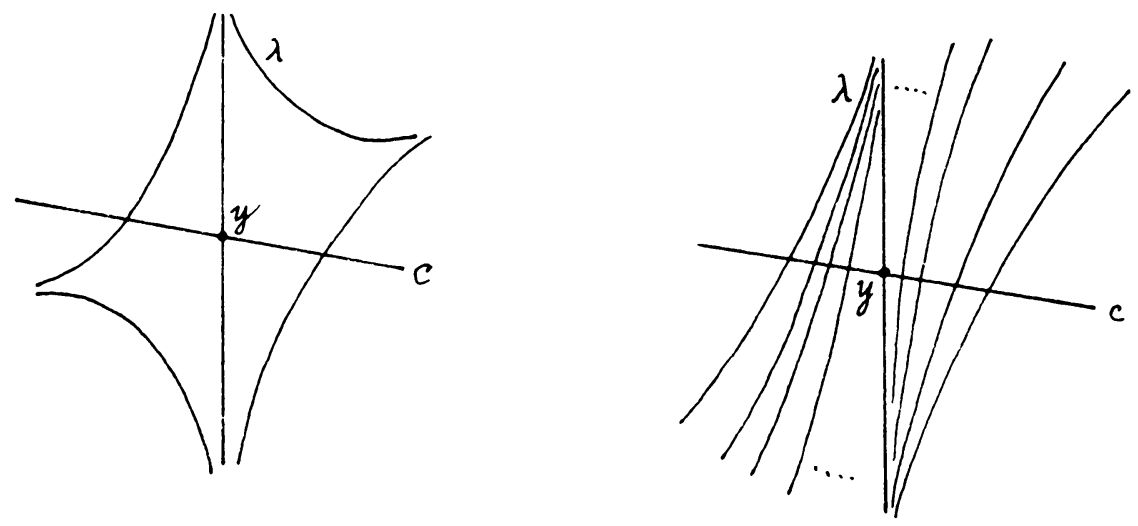

Figure 1. The two types of boundary intersections 
leaf on both sides. We call $y$ a boundary intersection if the direction of spiraling on the two sides is different. We now define the alternation number $a(\lambda, c)$ to be the number of boundary intersections. Notice that $a(\lambda, c)$ is always finite since the boundary intersection points are isolated.

We may similarly decide which points of $\lambda \cup c$ are boundary intersections of the support of a general current $c$ with $\lambda$, and define $a(\lambda, c)$ to be the total transverse $c$-measure of the set of boundary intersections. In fact,

Proposition 5.1. For a fixed finite-leaved maximal lamination $\lambda$ on a surface $S$, $a(\lambda, c)$ is a finite-valued continuous function on $\mathscr{C}(S)$.

Proof of 5.1. This proof is essentially a reiteration of the proof given in [37] for measured laminations. We will construct a continuous function $C(\lambda)$ on $\mathbf{P}(S)$ such that

$$
a(\lambda, c)=\int_{\mathbf{P}(S)} C(\lambda) d c
$$

for all $c \in \mathscr{C}(S)$.

Every isolated leaf $l$ of $\lambda$, lifted to the universal cover, separates two ideal triangles whose union forms an ideal quadrilateral. If a geodesic $m$ crosses $l$ it gives a boundary intersection if and only if it passes through opposite sides of the quadrilateral.

We now choose a particular continuous function defined on $[0,1]$ which is 0 at the endpoints and whose integral is 1 . By scaling this gives a continuous function defined on any interval whose integral is 1 and which is defined to be 0 at the endpoints.

For each quadrilateral defined by removing a leaf $l$, and for each geodesic which intersects opposite sides of that quadrilateral scale the above function to the intersection of the geodesic with the quadrilateral and lift to $\mathbf{P}\left(\mathbf{H}^{2}\right)$. We define $\bar{C}(\lambda)$ by adding up over all quadrilaterals and all geodesics (at each point of $\left.\mathbf{P}\left(\mathbf{H}^{2}\right)\right)$. Notice that at each point there are at most three contributions and that each contribution is continuous on its quadrilateral of definition, since as a leaf intersecting opposite sides of the quadrilateral approaches one that does not, the length of the interval goes to infinity. This function was defined equivariantly so it descends to a function $\bar{C}(\lambda)$ on $\mathbf{P}(S)$ with the desired properties.

If $\alpha$ is any closed leaf of $\lambda$ with leaves of $\lambda$ spiraling in opposite directions about it, we add to $\bar{C}(\lambda)$ a unit contribution for every geodesic which crosses $\alpha$, supported entirely on a small neighborhood of $\alpha$. The result, $C(\lambda)$, is still continuous.

Remark. Alternation number does not depend on the underlying hyperbolic structure on $S$.

5.2 Winding about the boundary. We now need to formalize what we mean when we say that $c$ does not wind too much about $\partial S$. Let $c$ be a current on $S$ which does not contain any component of $\partial S$. (Recall, that we have defined our currents to have their support within the convex core.) Let $\hat{\tau}_{0}$ be a hyperbolic metric of finite area on $S_{0}$, i.e. we have pinched all the funnels to cusps. Let $\left(S_{0}, \hat{\tau}_{0}\right)=\mathbf{H}^{2} / \widehat{\Gamma}$ and $\left(S_{0}, \tau_{0}\right)=\mathbf{H}^{2} / \Gamma$. In [14] Floyd showed that there is a map of $L_{\Gamma}$ to $L_{\widehat{\Gamma}}$ (induced by the topological identification of $S_{0}$ with itself) which is one-to-one except that it identifies the fixed points of 
elements of $\Gamma$ which are parabolic in $\widehat{\Gamma}$. So if $c \in \mathscr{C}(S)$ does not contain a component of $\partial S$ it is identified with a well-defined current $\hat{c} \in \mathscr{C}\left(S_{0}, \hat{\tau}_{0}\right)$.

To formalize what we mean by "winding too much about the boundary" we need to recall the thick-thin decomposition of a hyperbolic manifold. We recall that the injectivity radius of a hyperbolic $n$-manifold $N$ at a point $x$, denoted $\operatorname{inj}_{N}(x)$, is defined to be half of the length of the shortest (homotopically nontrivial) loop through $x$. There exists a constant $\mu_{n}$, called the Margulis constant, such that if $\varepsilon<\mu_{n}$ and

$$
N_{\text {thin }(\varepsilon)}=\{x \in N \mid \operatorname{inj}(x)<\varepsilon\}
$$

then every component of $N_{\operatorname{thin}(\varepsilon)}$ has abelian fundamental group (see Thurston [39] or Morgan [27]). If $n=2$ every component of $N_{\operatorname{thin}(\varepsilon)}$ is either a cusp (i.e. a horosphere in $\mathbf{H}^{2}$ modulo a parabolic action of $\mathbf{Z}$ ) or a cylindrical neighborhood of a closed geodesic. If $n=3$ every component of $N_{\operatorname{thin}(\varepsilon)}$ is either a cusp (i.e. a horoball in $\mathbf{H}^{3}$ modulo a parabolic action of either $\mathbf{Z}$ or $\mathbf{Z} \oplus \mathbf{Z})$ or a solid torus neighborhood of a geodesic.

We define

$$
\delta_{\partial S}(c)=\max \left\{\varepsilon \mid \hat{c} \in \widehat{S}_{\text {thick }(\varepsilon)}\right\} .
$$

(One could obtain more precise results by requiring that $\widehat{S}$ have no thin parts other than the cusps associated to components of $\partial S$, but this will not be necessary for our work.) It would seem that $\delta$ would depend strongly on the hyperbolic structure of $\widehat{S}$. However, the following lemma tells us that $\delta$ gives us a rough idea how far $c$ travels into the thin part associated to any component of $\partial S$ (if there is one) for any hyperbolic structure on $S$.

Lemma 5.2. If $\delta_{\partial S}(c)>\varepsilon$, then there exist $\varepsilon^{\prime}$ (depending only on $\varepsilon$ ) such that if $\tau$ is any hyperbolic structure on $S_{0}$ then the support of $c$ on $\left(S_{0}, \tau\right)$ never enters the interior of a component of $\left(S_{0}, \tau\right)_{\operatorname{thin}\left(\varepsilon^{\prime}\right)}$ corresponding to $\partial S$.

Proof of 5.2. We will first prove the lemma for all hyperbolic structures on $S$ in which each component of $\partial S$ corresponds to a cusp. We translate the statement $\delta_{\partial S}(c)>\varepsilon$ into a statement about lifts of leaves of $c$ in the universal cover. Roughly it means that there exists some $K>0$ such that if $l$ is the lift of any leaf of $c, \gamma$ is a parabolic curve corresponding to a component of $\partial S$, and $T$ is an isometry of $\mathbf{H}^{2}$ covering $\gamma$, then there exist at most $K T$-translates of $l$ 's rightmost endpoint between $l$ 's two endpoints. To see this normalize so that $T$ is $z \mapsto z+1$. How far $l$ enters the thin part is determined (exactly) by the (Euclidean) distance between the endpoints of $l$. This distance is determined up to the nearest integer by the ordering of the limit set (this may be measured by looking at how many of the translates of the attracting fixed point under $\langle z \mapsto z+1\rangle$ lie between the attracting and repelling fixed points). In particular, if there are $n$ translates between the two endpoints, then $l$ must enter the $\frac{2}{n}$ thin part, but it cannot enter the $\frac{2}{n+4}$-thin part. We recall that the ordering of the limit set is an invariant of the group, so the number of translates is completely determined by $l$ 's endpoints and does not depend on the hyperbolic structure. So given $\varepsilon \geq \frac{2}{N}$ we may choose $\varepsilon^{\prime}=\frac{2}{N+4}$.

If some component $\gamma$ of $\partial S$ is noncuspidal, we normalize so that $T(z)$ $=\lambda z($ where $\lambda>1)$ and such that $l$ 's leftmost endpoint lies at 1 . If $\delta_{\partial s}(c) \geq$ $\frac{2}{n-2}$, then at most $n-1 T$-translates of 1 lie between 1 and $l$ 's rightmost 
endpoint. Thus, $l$ 's rightmost endpoint lies between 1 and $\lambda^{n}$. The distance a point is translated by $T$ is determined entirely by its (hyperbolic) distance to the $y$-axis. No point on $l$ is closer to the $y$-axis than $r(\lambda, n)=1+$ $\left(\lambda^{n}-1\right) i / 2$, so every point on $l$ is translated (by $T$ ) a distance less than $d(\lambda, n)=d(r(\lambda, n), \lambda r(\lambda, n))$. But (by using formulas in $\S 7.2$ of Beardon [2]) we see that

$$
\cosh d(\lambda, n)=1+\frac{(\lambda-1)^{2}\left(\lambda^{2 n}-2 \lambda^{n}+5\right)}{2 \lambda\left(\lambda^{2 n}-2 \lambda^{n}+1\right)} .
$$

We now observe that

$$
\lim _{\lambda \mapsto 1} \cosh d(\lambda, n)=1+\frac{2}{n^{2}} .
$$

Thus we can find $\phi(n)$ (depending only on $n$ ) such that if $\lambda \leq e^{\phi(n)}$, then $d(\lambda, n) \geq \phi(n)$. Thus every point on $l$ is translated a distance greater than $\phi(n)$. Therefore if $\delta_{\partial S}(c) \geq \frac{2}{n-2}$, we may choose $\varepsilon^{\prime}$ to be $\min \left\{\phi(n), \frac{2}{n+2}\right\}$.

We also need to know that every current $c$ with $\delta_{\partial S}(c)>\varepsilon$ may be approximated by a sequence of currents $c_{i}$ which are linear combinations of closed curves with $\delta_{\partial S}\left(c_{i}\right)>\varepsilon$. But if one reviews the proof that closed curves are dense in $\mathscr{C}(S)$ (see for example the proof in Bonahon [9] or [6]), the first step produces a linear combination of closed curves which are near to $c$ in the Hausdorff topology on supports as well as in the current space. We state this as:

Lemma 5.3. Any current $c \in \mathscr{C}(S)$ such that $\delta_{\partial S}(c)>\varepsilon$ may be approximated by currents $c_{i}$ which have as support a finite collection of closed geodesics and such that $\delta_{\partial S}\left(c_{i}\right)>\varepsilon$ for all $i$.

5.3 Efficiency achieved. We are now prepared to state efficiency of pleated surfaces formally.

Proposition 5.4. Let $\rho: F_{g} \rightarrow \mathrm{PSL}_{2}(\mathbf{C})$ be a Schottky group such that $N=$ $\mathbf{H}^{3} / \rho\left(F_{g}\right)$ is homeomorphic to the interior of $S \times I$. If $p:(S, \tau) \rightarrow N$ is a pleated surface (homotopic to $S \times\left\{\frac{1}{2}\right\}$ ) with a maximal finite leaved pleating locus $\lambda$ whose only closed leaves are components of the boundary, such that $l_{N}(\partial S) \leq B$ and $c$ is any current on $S$ with $\delta_{\partial S}(c)>\varepsilon$, then there exists a constant $C>0$ (depending only on $\varepsilon, B$, and the topological type of $S$ ) such that

$$
l_{N}(c) \leq l_{\tau}(c) \leq l_{N}(c)+C a(c, \lambda) .
$$

Remark. (1) The restriction that $c$ not wind too much about $\partial S$ is necessary since if some component of $\partial S$ is homotopic to a thin part, going deep into the thin part, traveling up the axis and then coming straight back out (which is approximately what the representative of a curve which winds a lot about the component does in the pleated surface) may be arbitrarily less efficient than traveling across the boundary of the thin part. This phenomenon occurs when we have a cyclic loxodromic subgroup which is geometrically close to a parabolic group of rank 2. (See the discussion of this phenomenon in Thurston [39] or Jorgensen-Marden [20].)

(2) The proof works equally well for any pleated surface $p: S \rightarrow N$ (into any hyperbolic 3-manifold $N$ ) with a maximal finite-leaved pleating locus $\lambda$ such that $(S, \partial S, p)$ is doubly incompressible (see below). 
Proof of 5.4. The proof is virtually the same as in Thurston's proof of efficiency of pleated surfaces in quasi-Fuchsian groups (see [37]). As this proof has not been published we will give a self-contained proof. We will follow Thurston's proof taking care to point out the (very few) places where the proof differs. We will also give a longer exposition of Thurston's use of the relative uniform injectivity theorem in hopes of clarifying matters.

Notice that it is only necessary to prove the statement of the lemma for closed curves, since any current with $\delta_{\partial S}(c)>\varepsilon$ may be approximated by finite unions of closed curves with the same property and length is continuous on $\mathscr{C}(N)$ and alternation number is continuous on $\mathscr{C}(S)$ (which is identified with $\mathscr{C}(N)$ ).

The main tool is the relative uniform injectivity theorem [38]. (The proof of the relative uniform injectivity theorem is a straightforward generalization of the proof of the uniform injectivity theorem in [36].) We first must recall the definition of a doubly incompressible map. Let $N$ be a 3-manifold, $f: S \rightarrow N$ a map of a compact surface (possibly with boundary) into $N$, and $X \subset S$ an (embedded) system of nontrivial homotopically distinct simple closed curves on $S$ including all components of $\partial S$. Then $(S, X, f)$ is said to be doubly incompressible if

(a) there are no essential immersed annuli with boundary in $S-X$,

(b) any compressible curve in $S$ intersects $X$ at least three times (i.e. if $\gamma$ is any homotopically nontrivial curve on $S$ such that $f(\gamma)$ is homotopically trivial in $N$ then $\gamma$ intersects $X$ at least three times), and

(c) every maximal abelian subgroup of $\pi_{1}(S-X)$ is mapped to a maximal abelian subgroup of $\pi_{1}(N)$.

(A mapping $h:(A, \partial A) \rightarrow(N, f(S))$ is said to be an essential immersed annulus if $\partial h: \partial A \rightarrow f(S)$ can be written as $f \circ \partial h_{0}$ where $\partial h_{0}: \partial A \rightarrow S$, and $h$ cannot be homotoped by homotopy of pairs $H$ to a map $h^{\prime}:(A, \partial A) \rightarrow$ $(N, f(S))$ in such a way that $\partial H: \partial A \times I \rightarrow f(S)$ can be written as $f \circ \partial H_{0}$ where $\partial H_{0}: \partial A \times I \rightarrow S$.)

Notice that $(S, \partial S, p)$ is doubly incompressible.

Theorem 5.5 (Thurston [38]). Let $S$ be a compact surface, and $X$ a collection of nontrivial, homotopically distinct simple closed curves on $S$ which includes all boundary components. Let $B$ and $\varepsilon_{0}$ be positive constants. Among all pleated surfaces $p:(S, \tau) \rightarrow N(N$ a variable hyperbolic manifold $)$ pleated along laminations $\lambda$ containing $X$, where $(S, X, f)$ is doubly incompressible and the total length of $X$ in $N$ is less than $B$, the associated projective maps $P: \lambda \rightarrow \mathbf{P}(N)$ are uniformly injective on the $\varepsilon_{0}$-thick part of $S$. That is for every $\varepsilon_{1}>0$ there is $a \delta 0$ such that for any such $\tau, N, \lambda$ and $p$ and for any two points $x$ and $y \in \lambda$ whose injectivity radius is greater than $\varepsilon_{0}$, if $d(x, y) \geq \varepsilon_{1}$ then $d(P(x), P(y)) \geq \delta$.

Since $c$ does not contain a closed leaf of $\lambda$, we know that $a(\lambda, c)>0$ and there is a chain of length $a(\lambda, c)$ consisting of leaves of $\lambda$ on which there are boundary intersections with $c$. We may then form a piecewise geodesic curve in the homotopy class of $c$ consisting of $a(\lambda, c)$ segments of $\lambda$ and $a(\lambda, c)$ “jumps" across $S-\lambda$. We now describe Thurston's algorithm for doing this efficiently.

Let $R$ be a strip of constant width, Thurston uses .5 , about $\tilde{c}$ in $(\widetilde{S}, \tilde{\tau}) \cong$ $\mathbf{H}^{2}$. By orienting $\tilde{c}$ we may obtain a notion of whether a point in $x \in R$ lies 


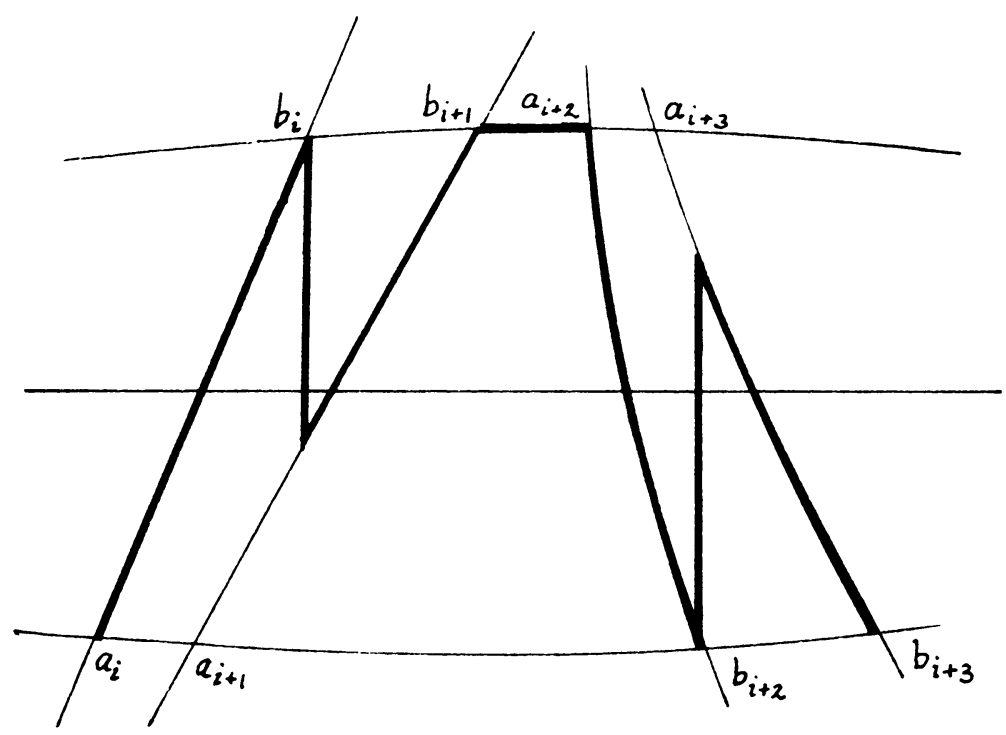

FIGURE 2. A picture of the polygonal approximation $\tilde{m}$ in the proof of Proposition 5.4.

to the left or right of a point $y \in R$. Since every point in $R$ lies on a geodesic segment of length one perpendicular to $\tilde{c}$, we may say that $x$ lies to the right of $y$ if the geodesic segment $x$ lies on is to the right of the geodesic segment $y$ lies on. The leaves of $\lambda$ passing through the boundary intersection points of $\lambda$ with $c$ give us an infinite chain of geodesics $g_{i}, i=[-\infty \cdots \infty]$, connecting the two endpoints of $\tilde{c}$, with endpoints of successive geodesics meeting at $S_{\infty}^{1}$. Let $a_{i}$ be the leftmost endpoint of $R \cap g_{i}$ and $b_{i}$ the rightmost (if $a_{i}$ and $b_{i}$ lie on the same geodesic segment perpendicular to $\tilde{c}$, we arbitrarily choose one endpoint to be leftmost and the other rightmost). Let $A_{i}$ and $B_{i}$ be the (hyperbolic) perpendicular projections of $a_{i}$ and $b_{i}$ to $\tilde{c}$. Then $A_{i}$ lies to the left of $A_{i+1}, B_{i}$ lies to the left of $B_{i+1}$. Moreover, if $A_{i+1}$ lies to the right of $B_{i}$ the distance between them is bounded from above by an a priori constant $C_{0}$.

If $B_{i}$ lies to the right of $A_{i+1}$, let $x_{i+1}$ be the point on $g_{i+1}$ whose perpendicular projection to $\tilde{c}$ is $B_{i}$, otherwise let $x_{i+1}=a_{i+1}$. (This is to make sure that we always make forward progress in $R$.)

Now define a polygonal path $\tilde{m} \subset \mathbf{H}^{2}$ to consist of the geodesic segments

$$
\ldots \overline{b_{i-1} x_{i}} \cdot \overline{x_{i} b_{i}} \ldots
$$

$\overline{x_{i} b_{i}}$ lies on $\lambda$ and $\overline{b_{i-1} x_{i}}$ is a "jump" of length at most $1+C_{0}$ and the length of $\overline{b_{i-1} x_{i}} \cdot \overline{b_{i} x_{i}}$ is at most $2+C_{0}$ more than the distance from $B_{i}$ to $B_{i+1}$. Everything is defined equivariantly so $\tilde{m}$ projects to a polygonal curve $m$ on $(S, \tau)$. $m$ consists of $a(\lambda, c)$ segments on leaves of $\lambda$ plus $a(\lambda, c)$ "jumps" from one leaf to another of length $\leq 1+C_{0}$. Moreover,

$$
l_{\tau}(c) \leq l_{\tau}(m) \leq l_{\tau}(c)+C_{1} a(\lambda, c)
$$

where $C_{1}=2+C_{0}$. See Figure 2 .

In order to be able to make use of the relative uniform injectivity theorem we need to alter $m$ so that it makes no jumps in $(S, \tau)_{\operatorname{thin}\left(\varepsilon^{\prime}\right)}$, where $\varepsilon^{\prime}$ is 
associated to $\varepsilon$ as in Lemma 5.2. First notice that $m$ does not enter any $\varepsilon^{\prime}$ thin parts corresponding to components of $\partial S$, by the above assumption on $\delta_{\partial S}(c)$. Thus any time $m$ enters a component of $(S, \tau)_{\operatorname{thin}\left(\varepsilon^{\prime}\right)}$ either it winds about and comes out the side it came in or it winds about and goes through. (The main technical difference between our proof and Thurston's is that we have to allow the possibility that $m$ enters a thin part winds about and comes out the same side, this forced us to introduce $\delta_{\partial S}(c)$ to measure how deep $m$ may enter a thin part corresponding to a component of $\partial S$.)

If it winds about and comes out the same side we project the whole picture onto the boundary of the component of $(S, \tau)_{\operatorname{thin}\left(\varepsilon^{\prime}\right)}$. If it goes through we project all the jumps onto one boundary of the component of $(S, \tau)_{\operatorname{thin}\left(\varepsilon^{\prime}\right)}$ (i.e. we make sure that it makes all its jumps either right before entering the thin part or right after leaving the thin part). We then form a new polygonal curve $n$ by homotoping each of the new jumps to a geodesic arc, without moving the endpoints. Notice that no jump of our new polygonal curve $n$ may wind more than once about the boundary of the component of $(S, \tau)_{\operatorname{thin}\left(\varepsilon^{\prime}\right)}$, so it has length at most some constant $R$, where $R$ may be taken to be the maximal length of any boundary component of $(S, \tau)_{\operatorname{thin}\left(\varepsilon^{\prime}\right)}$. Moreover, at each stage we have increased the length by at most $R$ times the number of jumps.

To summarize, we have now produced a polygonal curve $n$ on $(S, \tau)$ which consists of at most $a(\lambda, c)$ segments on leaves of $\lambda$, and at most $a(\lambda, c)$ jumps of length at most $K=\max \left\{1+C_{0}, R\right\}$ all of which have their endpoints in the $\varepsilon^{\prime}$-thick part of $(S, \tau)$. Notice that $n$ is also polygonal in $N$ and that

$$
l_{\tau}(c) \leq l_{N}(n) \leq l_{\tau}(c)+C_{2} a(\lambda, c)
$$

where $C_{2}=2+C_{0}+R$.

We now construct a pleated annulus $A$ realizing the homotopy between $n$ and the geodesic $c^{*}$ in $N$ in the free homotopy class of $c$, by straightening and spinning a triangulation of the homotopy between $c^{*}$ and $n$. The resulting annulus has area less than $2 \pi a(\lambda, c)$, since it is made up of $2 a(\lambda, c)$ hyperbolic triangles. (We could also have made use of simplicial hyperbolic annuli as in [7].) We will cut $n$ up into pieces and use hyperbolic geometry and the relative uniform injectivity theorem to control the length of each of the pieces by $l_{N}\left(c^{*}\right)$ and $a(\lambda, c)$. This formulation of the argument is reminiscent of similar arguments in Bonahon [7].

Let $K=\max \left\{1+C_{0}, R\right\}$ be as above. We now choose $D \leq K$ with the following two properties:

(D1) Let $\alpha, \beta$, and $\gamma$ be any 3 nonintersecting geodesic $\operatorname{arcs}$ in $\mathbf{H}^{2}$ of length at least $K$ and $x \in \alpha, y \in \beta$, and $z \in \gamma$ all lie at least a distance $\frac{K}{2}$ away from either endpoint of the arc they lie on. Then $d(x, y) \leq 2 D$, implies $d(x, z) \geq 2 D$.

(D2) Let $\alpha$ and $\beta$ be any two geodesic arcs in $\mathbf{H}^{3}$ of length at least $K$ in $\mathbf{H}^{3}$, such that if $x \in \alpha$ then there exists $y \in \beta$ such that $d(x, y) \leq$ $D$. If $d(x, y) \leq D$, then $d(P(x), P(y))<\delta$ where $P: \alpha \cup \beta \rightarrow \mathbf{P}\left(\mathbf{H}^{3}\right)$ is the associated projective map and $\delta$ is obtained from the relative uniform injectivity theorem by taking both $\varepsilon_{0}$ and $\varepsilon_{1}$ to be $\varepsilon^{\prime}$.

We will say that a point $x \in n$ is in $n_{0}$ if it lies within $\frac{K}{2}$ of one of the endpoints of the geodesic arc it lies within. Notice that every "jump" of $n$ is contained entirely within $n_{0}$ and that there are at most $a(\lambda, c)$ components of 
$n_{0}$. Moreover,

$$
l\left(n_{0}\right) \leq 2 K a(\lambda, c) .
$$

We will say that a point $x \in\left(n-n_{0}\right)$ is in $n_{1}$ if there exists a vertex $v$ of $n$ such that $d(x, v) \leq D$ where distance is measured in the intrinsic hyperbolic metric on $A$. Notice that, by property (D1), that each vertex lies within $D$ of at most two segments of $n-n_{0}$ and that the portion of each such segment which lies within $D$ of $v$ has length $\leq 2 D$. Therefore there are at most $4 a(\lambda, c)$ components of $n_{1}$ and thus

$$
l\left(n_{1}\right) \leq 8 D a(\lambda, C) .
$$

We will further say that a point $x \in n-\left(n_{0} \cup n_{1}\right)$ is in $n_{2}$ if the geodesic segment $p_{x}$ perpendicular to $n$ beginning at $x$ (and ending when it first reaches $\partial A$ ) has length $\geq D$. Let $R_{2}$ denote the set of points which lie on a geodesic segment $p_{x}$ perpendicular to $n$ and originating at a point $x \in n_{2}$. Since any point in $R_{2}$ lies within $D$ of at most 2 points in $n_{2}$, we see that $\operatorname{area}\left(R_{2}\right) \geq$ $l\left(n_{2}\right) \frac{D}{2}$. Thus,

$$
l\left(n_{2}\right) \leq \frac{2 \operatorname{area}(A)}{D} \leq \frac{4 \pi}{D} a(\lambda, c) .
$$

Let $\alpha$ be a component of $n-\left(n_{0} \cup n_{1} \cup n_{2}\right)$. We will say that $y \in d(\alpha)$ if there exists $x \in \alpha$ such that the geodesic segment $p_{x}$ perpendicular to $n$ and originating at $x$ terminates at $y$. By construction $d(\alpha)$ lies entirely on one segment of $\partial A$ (as it contains no vertices). Moreover, let $R_{\alpha}$ denote the set of points in $A$ which lie on geodesic segments perpendicular to $n$ and originating in $\alpha$. Notice that, by property (D1), no point of $A$ lies in more than two such regions. If one of $\alpha$ 's endpoints $x$ lies in $n_{2}$ then $p_{x}$ has length $D$ and $R_{\alpha}$ contains a right triangle whose two shorter sides have length $D$ and $l(\alpha)$. Since there are at most $5 a(\lambda, c)$ components of $n_{0} \cup n_{1}$, there are at most $5 a(\lambda, c)$ components of $n-\left(n_{0} \cup n_{1} \cup n_{2}\right)$ with both endpoints on $n_{0} \cup n_{1}$.

Let $n_{3}$ denote the set of components of $n-\left(n_{0} \cup n_{1} \cup n_{2}\right)$ which have length $\leq K$. If $\alpha$ is a component of $n_{3}$ with at least one endpoint on $n_{2}$, then $R_{\alpha}$ has area at least as great as the right triangle with sides $D$ and $l(\alpha)$, in particular there exists $C_{3}$ such that area $\left(R_{\alpha}\right) \geq C_{3} l(\alpha)$. Also, recall that there are at most $5 a(\lambda, c)$ components of $n_{3}$ with both endpoints on $n_{0} \cup n_{1}$. Thus,

$$
l\left(n_{3}\right) \leq \frac{\operatorname{area}(A)}{2 C_{3}}+5 K a(\lambda, c) \leq\left(\frac{2 \pi}{2 C_{3}}+5 K\right) a(\lambda, c) .
$$

If $\alpha$ is a component of $n-\left(n_{0} \cup n_{1} \cup n_{2} \cup n_{3}\right)$ with at least one endpoint in $n_{2}$ then the area of $R_{\alpha}$ is greater than the area $G$ of the right triangle with sides $D$ and $K$, so there are at most $C_{4} a(\lambda, c)$ components of $n-\left(n_{0} \cup n_{1} \cup n_{2} \cup n_{3}\right)$ where $C_{4}=\frac{2 \pi}{2 G}+5$. See Figure 3 .

Let $n_{4}$ denote the collection of components $\alpha$ of $n-\left(n_{0} \cup n_{1} \cup n_{2} \cup n_{3}\right)$ such that $d(\alpha)$ lies in $n$. Let $\alpha$ be a component of $n_{4}, x_{1}$ and $x_{2}$ be the endpoints of $\alpha$, and $y_{1}$ and $y_{2}$ be the endpoints of $d(\alpha)$. Recall that by property (D2) that $d\left(P\left(x_{i}\right), P\left(y_{i}\right)\right)<\delta$ for both $i=1$ and $i=2$. Since $p: S \rightarrow N$ is doubly incompressible the set of components of $\widetilde{S}_{\operatorname{thin}\left(\varepsilon^{\prime}\right)}$ maps injectively into the components of $\tilde{N}_{\text {thin }\left(\varepsilon^{\prime}\right)}$, so both $\alpha$ and $d(\alpha)$ are contained entirely within $S_{\left.\text {thick( } \varepsilon^{\prime}\right)}$. Therefore, by the relative uniform injectivity theorem, there exists a path $\gamma_{i}$ on $S$ of length $\varepsilon^{\prime}$ joining $x_{i}$ to $y_{i}$, and a portion $r_{i}$ of $n$ such 


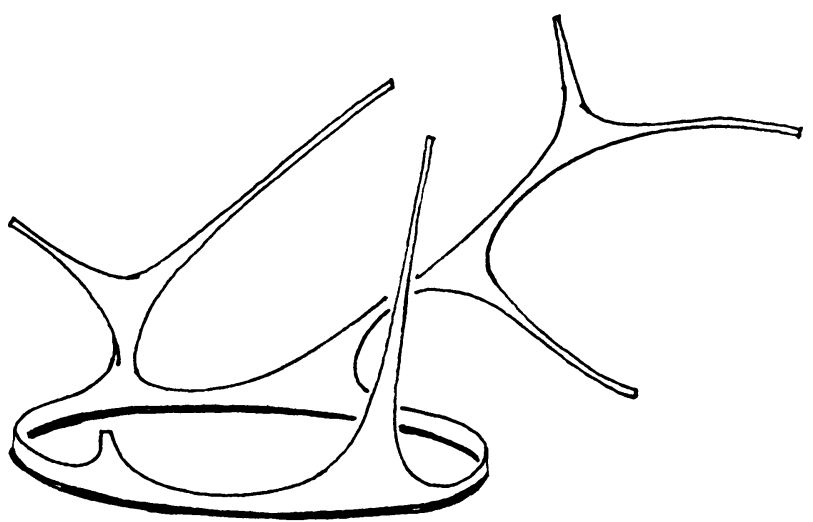

FIGURE 3. The pleated annulus $A$, nearly parallel portions of $n$ look like "crowns" and produce shortenings of $n$ in $S$.

that $r_{i} \cup \gamma_{i}$ bounds a disk on $S$ (for both $i=1$ and $i=2$ ). Now either $r_{1}$ or $r_{2}$ contains both $\alpha$ and $d(\alpha)$, so we may assume that $r_{1}$ does. Then $\gamma_{1}$ may be used to shorten the length of $n$ on $S$ by at least $2 l(\alpha)-\varepsilon^{\prime}$. But since $l(n)-l\left(c^{*}\right) \leq C_{2} a(\lambda, c)$ and there are at most $C_{4} a(\lambda, C)$ components of $n_{4}$,

$$
l\left(n_{4}\right) \leq \frac{C_{2}}{2} a(\lambda, c)+\varepsilon^{\prime} C_{4} a(\lambda, c) .
$$

Let $n_{5}$ denote $n-\left(n_{0} \cup n_{1} \cup n_{2} \cup n_{3} \cup n_{4}\right)$. If $\alpha$ is a component of $n_{5}$, then $d(\alpha) \subset c^{*}$ and $l(d(\alpha)) \geq l(\alpha)-2 D$. So if $c_{\text {good }}$ denotes the portion of $c^{*}$ which lies in $d(\alpha)$ for some component $\alpha$ of $n_{5}$, then

$$
l\left(c_{\text {good }}\right) \geq l\left(n_{5}\right)-2 D C_{4} a(\lambda, c) .
$$

Now bringing all this together,

$$
\begin{aligned}
l\left(n_{5}\right)= & l(n)-l\left(n_{0}\right)-l\left(n_{1}\right)-l\left(n_{2}\right)-l\left(n_{3}\right)-l\left(n_{4}\right) \\
\geq & l(n)-2 K a(\lambda, c)-8 D a(\lambda, c)-\frac{4 \pi}{D} a(\lambda, c) \\
& -\left(\frac{2 \pi}{C_{3}}+5 K\right) a(\lambda, c)-\left(\frac{C_{2}}{2}+\varepsilon^{\prime} C_{4}\right) a(\lambda, c) \\
\geq & l(n)-C_{5} a(\lambda, c)
\end{aligned}
$$

(for some constant $C_{5}$ ). Therefore,

$$
\begin{aligned}
l\left(c^{*}\right) & \geq l\left(c_{\text {good }}\right) \geq l\left(n_{5}\right)-2 D C_{4} a(\lambda, c) \\
& \geq l(n)-C_{5} a(\lambda, c)-2 D C_{4} a(\lambda, c)
\end{aligned}
$$

which establishes our claim.

\section{THE PROOF}

We are now in a position to finish the proof of our main theorem.

Proof of Main Theorem. Extend $\partial S$ to a maximal lamination $\lambda$ on $S$, by adding finitely many infinite geodesics which spiral, at each end, about some 
component of $\partial S$. Let $p_{i}:\left(S, \tau_{i}\right) \rightarrow N$ be the pleated surface in $N_{i}$ with pleating locus $\lambda$ (where $\tau_{i}$ is the hyperbolic structure on $S$ induced by pleating along $\lambda$ in $N_{i}$ ).

Recall from Corollary 3.5 that there exists a sequence of currents $c_{i}=F\left(\mu_{i}\right)$ converging to a Masur domain current $c=F(\mu)$ such that $l_{N_{i}}\left(c_{i}\right)$ converges to 0 . We claim that there exists some $\varepsilon$ such that $\delta_{\partial S}\left(c_{i}\right)>\varepsilon$ for all $i$. If not, the geometric limit of the supports of $c_{i}$ would contain some component of $(\partial S)^{*}$, but then the geometric limit of the supports of $\mu_{i}$ would contain a closed leaf homotopic (in $H_{g}$ ) to a component of $\partial S$. But the geometric limit of the supports of $\mu_{i}$ contains the support of $\mu$, thus $\mu$ may be extended to a measured lamination $\mu^{\prime}$ whose support contains a closed leaf homotopic to a component of $\partial S$ by simply putting the counting measure on the closed leaf. But then $\mu^{\prime}$ would lie in the Masur domain, since $\mu$ does, which would contradict Lemma 4.2.

Now by Proposition 5.4,

$$
l_{\tau_{i}}\left(c_{i}\right) \leq l_{N_{i}}\left(c_{i}\right)+C a\left(\lambda, c_{i}\right) .
$$

Thus, by Proposition 4.6, $\left\{\tau_{i}\right\}$ converges (up to subsequence) in the Fricke space $\widehat{\mathscr{T}}(S)$. But since $S$ carries the entire homotopy, this implies that $\left\{\rho_{i}\right\}$ converges (up to subsequence) in $A H\left(H_{g}\right)$. More explicitly, we may think of $\rho_{i}$ as a representation $\rho_{i}: \pi_{1}(S) \rightarrow \operatorname{Isom}_{+}\left(\mathbf{H}^{3}\right)$ and the hyperbolic structure $\tau_{i}$ determines a representation $\hat{\rho}_{i}: \pi_{1}(S) \rightarrow \operatorname{Isom}_{+}\left(\mathbf{H}^{2}\right)$. Let $\tilde{p}_{i}: \mathbf{H}^{2} \rightarrow \mathbf{H}^{3}$ cover $p_{i}$, the fact that $\tilde{p}_{i}$ is an equivariant isometry implies that

$$
d_{\mathbf{H}^{3}}\left(\rho_{i}(\gamma)\left(\tilde{p}_{i}(x)\right), \tilde{p}_{i}(x)\right) \leq d_{\mathbf{H}^{2}}\left(\hat{\rho}_{i}(\gamma)(x), x\right)
$$

for all $\gamma \in \pi_{1}(S)$ and any $x \in \mathbf{H}^{2}$. The fact that we may find a convergent subsequence $\left\{\hat{\rho}_{j}\right\}$ of $\left\{\hat{\rho}_{i}\right\}$, implies that for any finite set of generators $\left\{\gamma_{k}\right\}$ of $\pi_{1}(S)$ and any point $x \in \mathbf{H}^{2}$ there exists $K$ such that

$$
d_{\mathbf{H}^{2}}\left(\hat{\rho}_{j}\left(\gamma_{k}\right)(x), x\right) \leq K
$$

for all $j$ and $k$. Therefore,

$$
d_{\mathbf{H}^{3}}\left(\rho_{j}\left(\gamma_{k}\right)\left(\tilde{p}_{j}(x)\right), \tilde{p}_{j}(x)\right) \leq K
$$

for all $j$ and $k$, which implies that we may choose a subsequence of $\rho_{j}$ which converges. (Recall that we are at each stage allowed to normalize the picture so that $p_{j}(x)$ lies at the origin in the Poincare ball model for $\mathbf{H}^{3}$.)

We may also state a version of our result which has purely internal assumptions.

Theorem 6.1. Let $\left\{\rho_{i}\right\}$ be a sequence of Schottky groups. If $H_{g}=S \times I$, and $c$ is a binding current on $S$, such that $\delta_{\partial S}(c)>\varepsilon$ (for some $\varepsilon>0$ ), and $l_{N_{i}}(c) \leq K$ and $l_{N_{i}}\left((\partial S)^{*}\right) \leq K$ for all $i$ and some $K>0$, then $\left\{\rho_{i}\right\}$ has a convergent subsequence in $A H\left(H_{g}\right)$.

This entire discussion can be formulated in the language of R-trees. Given a divergent sequence in $A H\left(H_{g}\right)$, we may choose a subsequence whose limit may be thought of as an action (by isometries) of $F_{g}$ on an R-tree (see MorganShalen [29]). If, in this sequence, the traces of the representatives of the generators of $\pi_{1}(\partial S)$ are bounded, the resulting action is dual to a measured lamination on $S$ (see Morgan-Otal [28] or Skora [35]). Bestvina [5] and Paulin 
[34] have shown that this tree arises as the geometric limit of the associated hyperbolic manifolds with appropriate scaling constants (which converge to zero). We may interpret this as saying that the length (in $N$ ) of any current (on $S$ ) which intersects the limiting measured lamination also goes to infinity. (To make this interpretation rigorous requires, I think, something analogous to our efficiency of pleated surfaces.) Thus, no Masur domain lamination can have bounded length over the entire sequence.

Using this approach J. P. Otal has proved Thurston's conjecture for Schottky groups of genus 2 . He uses the fact that actions of the free group on two generators on R-trees are particularly well understood.

\section{REFERENCES}

1. W. Abikoff, The real analytic theory of Teichmüller space, Lecture Notes in Math., vol. 820, Springer-Verlag, 1980.

2. A. F. Beardon, The geometry of discrete groups, Graduate Texts in Math., vol. 91, SpringerVerlag, 1983.

3. L. Bers, Spaces of Kleinian groups, Maryland Conference in Several Complex Variables. I, Lectures Notes in Math., vol. 155, Springer-Verlag, 1970, pp. 9-34.

4. L. Bers and F. Gardiner, Fricke spaces, Adv. in Math. 62 (1986), 249-284.

5. M. Bestvina, Degenerations of hyperbolic space, Duke Math. J. 56 (1988), 143-161.

6. F. Bonahon, Bouts des variétés hyperboliques de dimension 3, prepublicationes de Orsay, 1985.

7. $\ldots$, Bouts des variétés hyperboliques de dimension 3, Ann. of Math. 124 (1986), pp. 71158.

8. __ The geometry of Teichmüller space via geodesic currents, Invent. Math. 92 (1988), 139-162.

9. __ Geodesic currents on hyperbolic groups, Arboreal Group Theory, edited by R. C. Alperin, Springer-Verlag, New York, 1991, pp. 143-168.

10. R. D. Canary, The Poincaré metric and a conformal version of a theorem of Thurston, Duke Math. J. 64 (1991), 349-359.

11. __ Hyperbolic structures on 3-manifolds with compressible boundary, Ph.D. thesis, Princeton Univ., 1989.

12. R. D. Canary, D. B. A. Epstein, and P. Green, Notes on notes of Thurston, Analytical and Geometrical Aspects of Hyperbolic Spaces, Cambridge Univ. Press, 1987, pp. 3-92.

13. A. Fathi, F. Laudenbach and V. Poenaru, Travaux de Thurston sur les surfaces, Asterique 66-67 (1979).

14. W. J. Floyd, Group completions and limit sets of Kleinian groups, Invent. Math. 57 (1980), 205-218.

15. __, Hyperbolic manifolds obtained by adding a 2-handle to a 3-manifold with compressible boundary, preprint.

16. C. McA. Gordon, On primitive sets of loops in the boundary of the handlebody, Topology Appl. 27 (1987), 285-299.

17. J. Hempel, 3-manifolds, Ann. of Math. Studies, no. 86, Princeton Univ. Press, 1976.

18. W. Jaco, Adding a 2-handle to a 3-manifold: an application to property $R$, Proc. Amer. Math. Soc. 92 (1984), 282-292.

19. T. Jorgensen, On discrete groups of Möbius transformations, Amer. J. Math. 98 (1976), 739-749.

20. T. Jorgensen and A. Marden, Algebraic and geometric convergence of Kleinian groups, Math. Scand. 66 (1990), 47-72.

21. S. P. Kerckhoff, The Nielsen realization problem, Ann. of Math. 117 (1983), 235-265. 
22. _ - The measure of the limit set of the handlebody group, Topology 29 (1990), 27-40.

23. I. Kra, On spaces of Kleinian groups, Comment. Math. Helv. 47 (1972), 53-69.

24. B. Maskit, Self-maps of Kleinian groups, Amer. J. Math. 93 (1971), 840-856.

25. __ Kleinian groups, Springer-Verlag, 1988.

26. H. Masur, Measured foliations and handlebodies, Ergodic Theory Dynamical Systems 6 (1986), 99-116.

27. J. W. Morgan, On Thurston's uniformization theorem for three-dimensional manifolds, The Smith Conjecture, edited by J. Morgan and H. Bass, Academic Press, 1984, pp. 37-125.

28. J. W. Morgan and J. P. Otal, Relative growth rates of closed geodesics on a surface under varying hyperbolic structures, preprint.

29. J. W. Morgan and P. Shalen, Valuations, trees and degenerations of hyperbolic structures. I, Ann. of Math. 120 (1984), 401-476.

30. __ Degeneration of hyperbolic structures II: Measured laminations in 3-manifolds, Ann. of Math. 127 (1988), 403-456.

31. __ Degeneration of hyperbolic structures III: Actions of 3-manifold groups on trees and Thurston's compactification theorem, Ann. of Math. 127 (1988), 457-519.

32. K. Ohshika, On limits of quasi-conformal deformations of Kleinian groups, Math. Z. 201 (1989), 167-176.

33. J. P. Otal, Courants geodesiques et produits libres, preprint.

34. F. Paulin, Topologie de Gromov équivariante, structures hyperboliques et arbres réels, Invent. Math. 94 (1988), 53-80.

35. R. Skora, Geometric actions of surface groups on $\Lambda$-trees, Comment. Math. Helv. 65 (1990), 519-533.

36. W. Thurston, Hyperbolic structures on 3-manifolds, I: Deformation of acylindrical manifolds, Ann. of Math. 124 (1986), 203-246.

37. __ Hyperbolic structures on 3-manifolds, II: Surface groups and 3-manifolds which fiber over the circle, preprint.

38. __ Hyperbolic structures on 3-manifold, III: Deformations of 3-manifolds with incompressible boundary, preprint.

39. __ The geometry and topology of 3-manifolds, lecture notes.

40. __ Minimal stretch maps between hyperbolic surfaces, preprint.

41. M. Wolf, The Teichmüller theory of harmonic maps, J. Differential Geometry 29 (1989), 449-479.

Department of Mathematics, Stanford University, Stanford, California 94305 48109

Current address: Department of Mathematics, University of Michigan, Ann Arbor, Michigan

E-mail address: canary@math.lsa.umich.edu 